\title{
Inflationary and dark energy regimes in $2+1$ dimensions
}

\author{
M. H. Christmann, F. P. Devecchi, G. M. Kremer and C. M. Zanetti \\ Departamento de Física, Universidade Federal do Paraná \\ Caixa Postal 19044, 81531-990, Curitiba, Brazil
}

December 24, 2018

\begin{abstract}
In this work we investigate the behavior of three-dimensional (3D) cosmological models. The simulation of inflationary and dark-energydominated eras are among the possible results in these 3D formulations; taking as starting point the results obtained by Cornish and Frankel. Motivated by those results, we investigate, first, the inflationary case where we consider a two-constituent cosmological fluid: the scalar field represents the hypothetical inflaton which is in gravitational interaction with a matter/radiation contribution. For the description of an old universe, it is possible to simulate its evolution starting with a matter dominated universe that faces a decelerated/accelerated transition due to the presence of the additional constituent (simulated by the scalar field or ruled by an exotic equation of state) that plays the role of dark energy. We obtain, through numerical analysis, the evolution in time of the scale factor, the acceleration, the energy densities, and the hydrostatic pressure of the constituents. The alternative scalar cosmology proposed by Cornish and Frankel is also under investigation in this work. In this case an inflationary model can be constructed when another non-polytropic equation of state ( the van der Waals equation) is used to simulate the behavior of an early $3 \mathrm{D}$ universe.
\end{abstract}

\section{Introduction}

The investigation of cosmological models in lower dimensions provide technical insights that can be applied to "realistic" models [1]. In the case of $2+1$ dimensions (3D models) several analysis were done mainly on the simulation of matter/radiation eras [3, 2]. 2D universes were also considered in the literature [6]: here, besides the matter/radiation periods, it was possible to simulate inflationary eras. In the case of homogeneous and isotropic models several alternative formulations were tested like the Jackiw-Teitelboim formulation $[\underline{6}$ in $2 \mathrm{D}$ and the scalar gravity model in $3 \mathrm{D}[\underline{3}$. In fact, in the $2 \mathrm{D}$ case the Einstein field 
equations are void of information and therefore the search for an alternative theory is mandatory [1].

One remarkable fact in 3D Einsteinian models is that the Riemann curvature tensor is zero outside sources, so there is no free propagation of the gravitational field in these formulations. As a consequence, the theories do not possess a Newtonian limit [3, 2. However, in a cosmological context the 3D space-time is supposed to be filled by the sources and in several cases a "regular" behavior follows. The basic ingredients in these models are the gravitational field equations (where the Einstein equations or alternative dynamics [1] are considered) the energy-momentum conservation law, the equations of state (in a macroscopic description) for the sources and their correspondent field equations, in a curved-space-time description.

In this work we extend the analysis done in [3, 2] considering the possibility of inflationary and dark energy regimes in $3 \mathrm{D}$ cosmologies. We make use of thermodynamics of relativistic gases and consider a two-constituents model with matter and a scalar field that is going to simulate the presence of the inflaton for a young universe or dark energy for an old universe. The simulation of interaction (through the gravitational field) between the constituents is done using a dynamical pressure term in the energy momentum-tensor of the sources. We obtain the evolution in time of the scale factor, the energy densities of the constituents and we focus mainly on the behavior of the acceleration of the expansion, as a fundamental ingredient to the classification of the cosmological regimes.

As we mentioned above another possibility suggested in the literature was the analysis of a 3D scalar gravity model 3 . Here we extend those results by using an exotic equation of state (the van der Waals equation (vdW), proposed for $4 \mathrm{D}$ cosmological models in [8] ) to simulate a three-eras regime that would correspond to an initial inflationary period, followed by a decelerated era where matter or radiation would dominate and finally an accelerated period dominated by dark energy represented by a cosmological constant.

The article is structured as follows. In section II we make a brief panorama of the physical principles involved in 3D cosmological models and in section III we present the analysis of a $3 \mathrm{D}$ inflationary period. In section IV we consider an old universe where the dark energy is represented by an scalar field or by the Chaplygin equation. In section $\mathrm{V}$ we show the analysis of the 3D scalar model. Finally in section VI we display our conclusions.

\section{Field equations and conservation laws}

In this section we make a brief review of the dynamics that rule the gravitational field and the sources in 3D space-times (for a detailed presentation see for instance [1, 3, 2]). One essential feature that appears in 3D is that the Riemann tensor components are zero outside sources [3, 2]. However, when we work in a cosmological context (taking a universe filled by sources), the 3D Einstein field equations permit the propagation of the gravitational field [3]: 


$$
R_{\mu \nu}-\frac{1}{2} R g_{\mu \nu}=-\kappa T_{\mu \nu}=-2 \pi G T_{\mu \nu},
$$

where $G$ is the "renormalized" gravitational constant for 3D space-time [3]. As in the $4 \mathrm{D}$ case, the hypotheses of isotropy and homogeneity are represented by the Robertson-Walker metric:

$$
d s^{2}=d t^{2}-a(t)^{2}\left[d r^{2}+r^{2} d \theta^{2}\right],
$$

where $a(t)$ is the scale factor. The sources of the gravitational field are seen as an out-of-equilibrium composite fluid. The correspondent energy-momentum tensor is given by

$$
T^{\mu \nu}=\left(\sum_{i}\left[\rho_{i}+p_{i}\right]+\varpi\right) U^{\mu} U^{\nu}-g^{\mu \nu}\left(\sum_{i} p_{i}+\varpi\right),
$$

where $\rho_{i}$ and $p_{i}(i=1,2, \ldots N)$ are the energy density and the hydrostatic pressure of the sources, respectively. $\varpi$ is the dynamical pressure, related to the viscosity of the cosmological fluid. When we work in a cosmological context and include the dynamical pressure it means that we are taking into account, phenomenologically, the interaction between the constituents through the gravitational field [5]. The energy-momentum tensor satisfies the correspondent conservation law $T_{; \nu}^{\mu \nu}=0$, that in a Co-moving frame reads

$$
\sum_{i} \dot{\rho}_{i}+2 \frac{\dot{a}}{a}\left\{\sum_{i}\left[\rho_{i}+p_{i}\right]+\varpi\right\}=0 .
$$

Solving the Einstein equations for the 3D Robertson-Walker metric we get

$$
H^{2}=\kappa \sum_{i} \rho_{i} \quad, \quad \frac{\ddot{a}}{a}=-\kappa\left(\sum_{i} p_{i}+\varpi\right) .
$$

where $H=\dot{a} / a$ is the Hubble function. In another possible case (such in the case of the dark energy) the sources can be modelled as a 3D scalar field $(\phi)$. This means that the curved space-time Klein-Gordon equation is necessary. In an isotropic and homogeneous $3 \mathrm{D}$ space-time it is given by

$$
\ddot{\phi}+2 H \dot{\phi}=-V^{\prime}(\phi)
$$

where $V$ is the potential and the exclusive dependence in time of $\phi$ is forced by the Robertson-Walker hypothesis.

Besides the 3D Einstein model other 3D gravitational theories has been proposed in the literature (such as the Einstein-Weyl model that includes torsion [1, 44 2]). In [3] was considered the possibility of an scalar 3D cosmology ruled by

$$
R=-2 \kappa T .
$$

This law gives in principle insufficient dynamical information for the gravitational field tensor. However, in Robertson-Walker cosmologies there is only one 
field to be determined (the scale factor $a(t)$ )and this makes possible to consider a model based on that scalar equation. In fact, in [3] a radiation dominated and a matter dominated 3D universe were simulated using the scalar cosmology. Another property of this model is that it permits a Newtonian limit [3].

In the following section we consider first the 3D Einstein theory to investigate the behavior of a young universe filled by a scalar field (representing the inflaton) and a matter constituent; both interacting through the gravitational field.

\section{Inflationary Universe in $3 \mathrm{D}$}

In this section we consider an inflationary universe. The main point here is to investigate how the 3D Einstein model describes the transition between an inflaton-dominated regime and the beginning of a matter era; focusing on the behavior of the acceleration $(\ddot{a})$ and on the energy densities $\left(\rho_{m}, \rho_{\phi}\right)$. The cosmological fluid is composed by two constituents: matter is represented by a barotropic equation of state and the inflaton is an scalar field $\phi(t)$. As it was explained in the last section the field equations and the energy-momentum conservation law furnish the dynamical relations between the observables. Taking the particular case of the energy conservation law it can be separated into two independent relations; here we are using the fact that the scalar field behavior is ruled by the curved space-time Klein-Gordon equation. The system of equations can be written in the following form

$$
\begin{gathered}
H^{2}=\kappa\left(\rho_{m}+\rho_{\phi}\right), \dot{H}+H^{2}=-\kappa\left(p_{m}+p_{\phi}+\varpi\right), \\
\dot{\rho}_{m}+2 H\left(\rho_{m}+p_{m}+\varpi\right)=0, \dot{\rho}_{\phi}+2 H\left(\rho_{\phi}+p_{\phi}\right)=0 .
\end{gathered}
$$

The thermodynamical behavior of both constituents is supposed to be ruled by 10

$$
\begin{gathered}
p_{m}=(\gamma-1) \rho_{m}, \quad p_{\phi}=(\nu-1) \rho_{\phi}, \\
\varpi=-\alpha\left(\rho_{m}+\rho_{\phi}\right) \Theta, \quad\left(\Theta=\nabla^{\mu} U_{\mu}=2 H\right),
\end{gathered}
$$

where $\alpha$ is a viscosity parameter and $\varpi$ is the dynamical pressure 5]. The combination of these expressions leads us to the following system

$$
\begin{gathered}
\frac{\rho_{m}}{\rho_{\phi}^{0}}=\left(1+\frac{\rho_{m}^{0}}{\rho_{\phi}^{0}}\right) H^{2}-\left(\frac{1}{a}\right)^{2 \nu}, \frac{\rho_{\phi}}{\rho_{\phi}^{0}}=\left(\frac{1}{a}\right)^{2 \nu} \\
\dot{H}=(2 \alpha H-\gamma) H^{2}+\frac{\gamma-\nu}{1+\rho_{m}^{0} / \rho_{\phi}^{0}}\left(\frac{1}{a}\right)^{2 \nu},
\end{gathered}
$$

where $\rho_{m}^{0} / \rho_{\phi}^{0}$ is the ratio between the energy densities at $t=0$. For convenience, we use the normalization $H^{2} \equiv \frac{H^{2}}{H_{0}^{2}}, H_{0}^{2}=\kappa\left(\rho_{m}^{0}+\rho_{\phi}^{0}\right)$ and $2 \alpha \equiv \alpha H_{0}$. The 


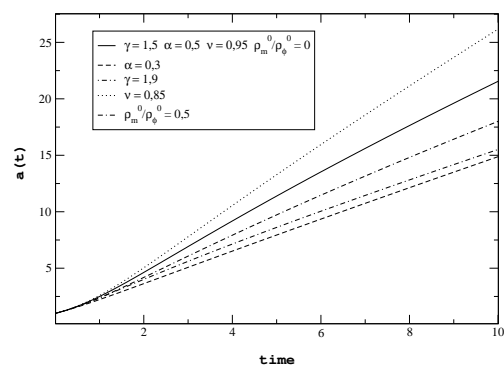

Figure 1: evolution in time for scale factor a(t) taking different values of the parameters

differential equation (13) is a non-linear, second order equation for the scale factor $a(t)$; it depends on four parameters $\gamma, \nu, \alpha$ and the quotient $\rho_{m}^{0} / \rho_{\phi}^{0}$. To solve this equation we must specify those values and two initial conditions $a(0)$ and $\dot{a}(0)$ and the intervals for the barotropic parameters. These are shown in the following table

\begin{tabular}{|c||c|}
\hline viscosity & $0<\alpha<1$ \\
\hline matter state equation & $1<\gamma<2$ \\
\hline inflaton state equation & $0<\nu<1$ \\
\hline \hline$a(0)$ & 1.0 \\
\hline$\dot{a}(0)$ & 1.0 \\
\hline$r=\rho_{m}^{0} / \rho_{\phi}^{0}$ & $0<r<0.5$ \\
\hline
\end{tabular}

The numerical analysis of the system (11-12) give the following results for the evolution of the physical quantities:

Figure 1 presents the behavior of the scale factor during the period of inflation. The numerical results show that an increasing viscosity $\alpha$ implies into a faster expansion. On the other hand larger values of $\gamma$ and $\nu$ (see the equations of state 10) furnish a slower expansion. Besides, for an increasing quotient $\rho_{m}^{0} / \rho_{\phi}^{0}$ the expansion is again slower.

In figure 2 we plot the behavior of acceleration function $\ddot{a}(t)$ for typical values of the parameters; the numerical analysis shows the transition of a strongly accelerated, expanding universe to a decelerated regime representing the end of the inflationary period and the beginning of a matter period. The results show that keeping the other parameters fixed the smaller the value of the viscosity $\alpha$ the smoother is the transition. On the other hand if we increase the ratio between the energy densities for $t=0$ we find again a smoother transition to a decelerated period.

In figure 3 we show the evolution in time of the energy densities. The representation of $\rho_{\phi}$ is in logarithmic scale. With the passing of time matter starts to predominate at the expense of the inflaton and gravitational field energies; this fact is in accord with the transition to a decelerated era as was shown in figure 2. 


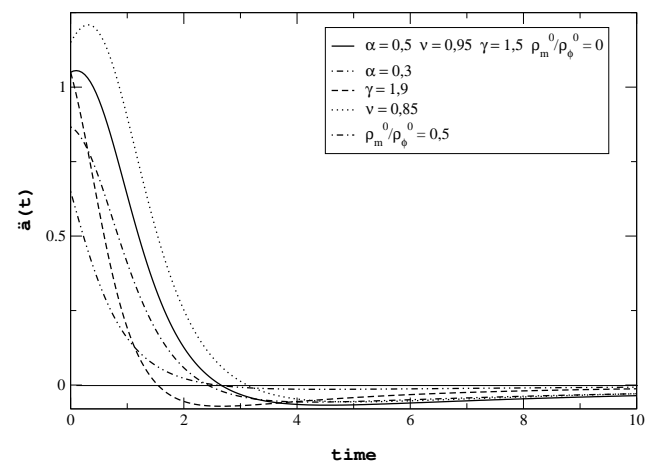

Figure 2: behavior of acceleration during inflation for different sets of parameters

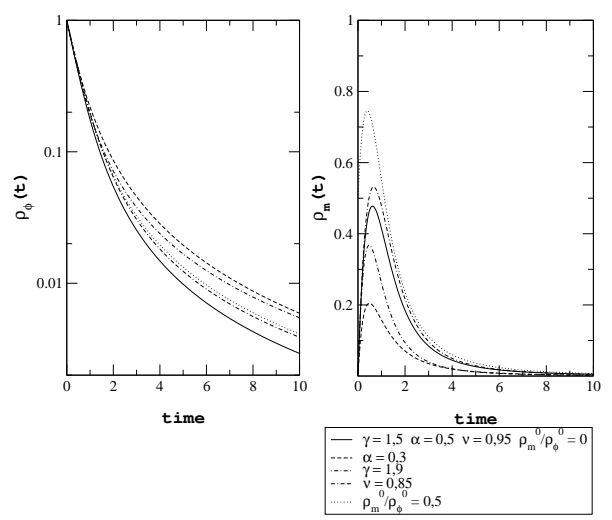

Figure 3: evolution in time of energy densities during the expansion in the inflationary regime 
It is worth mentioning that the quotient $\rho_{m}^{0} / \rho_{\phi}^{0}$ is essential to the classification of transitions: for the inflationary case we take a null (or small) value simulating that for $t=0$ we have a clear predominance of inflaton energy. For the dark-energy-dominated case ( as we will see in the following section) there is an opposite initial situation, and this is fundamental to obtain a transition between an initial decelerated period to a final accelerated era (as it is desired). These results show that the models give an adequate qualitative description of the cosmological regimes under analysis. Another important point is that in the case of the inflationary scenario the gravitational interaction between the constituents (represented by the dynamical pressure term) is relevant to the qualitative behavior of the acceleration.

On the other hand, the model also furnish solutions that do not describe transition regimes. For instance if the take the usual values of figure 2 for the parameters and a viscosity $\alpha \leq .29$ the transition does not occur, and the model only provides an ever decelerated universe.

\section{Accelerated 3D universe dominated by dark energy}

In this section we go forward in time putting an old 3D universe under investigation. Two cases are analyzed: i) the dark energy constituent modeled as an scalar field (for the original 4D model see [5]); ii) the dark energy is seen as a fluid ruled by the Chaplygin equation of state (proposed in $4 \mathrm{D}$ cosmological theories, see for instance [7]).

In the first case, as in the precedent section, the model considers the cosmological fluid consisting of a two-component gas with its energy-momentum tensor including a dynamical pressure term $(\varpi)$ to simulate the interaction between the constituents via the gravitational field. The dynamics are again ruled by

$$
\begin{gathered}
\dot{H}=(2 \alpha H-\gamma) H^{2}+\frac{\gamma-\nu}{1+\rho_{m}^{0} / \rho_{X}^{0}}\left(\frac{1}{a}\right)^{2 \nu}, \\
\frac{\rho_{m}}{\rho_{X}^{0}}=\left(1+\frac{\rho_{m}^{0}}{\rho_{X}^{0}}\right) H^{2}-\left(\frac{1}{a}\right)^{2 \nu}, \frac{\rho_{X}}{\rho_{X}^{0}}=\left(\frac{1}{a}\right)^{2 \nu} .
\end{gathered}
$$

as was mentioned in the last section the quotient $\rho_{m}^{0} / \rho_{X}^{0}$ defines the regime and in this case we are taking initial values that are compatible to the fact that matter dominates in the beginning of the era. The initial conditions and clocks are considered in a scale that is different from the previous section analysis; so the results are independent. As it is seen in figure 4 we still have a permanent expansion in this old universe. The results also show that an increasing initial ratio $\rho_{m}^{0} / \rho_{X}^{0}$ furnish a slower expansion. On the other hand only big changes 


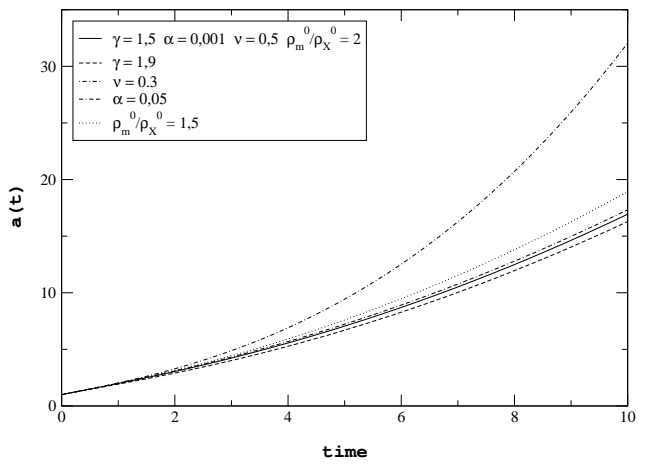

Figure 4: evolution in time of scale factor in the dark energy dominated regime

in the viscosity $\alpha$ give a substantial difference in the expansion rate. For an increasing $\gamma$ we have an slower expansion in this 3D old universe.

In figure 5 we plot the acceleration $\ddot{a}$ versus time; the results show that an initial period of deceleration is followed by an accelerated era. The extension of each period is related to the combination of parameters $\alpha, \nu, \gamma$ and the quotient between the initial energy densities. The results show that there is a critical value of $\nu$ (around 0.3) that implies in a fast growing of the acceleration. On the other hand, as it was expected, a larger quotient $\rho_{m}^{0} / \rho_{X}^{0}$ gives an earlier transition to the accelerated regime, indicating that the dark energy starts dominating the cosmological fluid, opening an era of eternal accelerated expansion. This behavior is qualitatively similar to the $4 \mathrm{D}$ case 7, [5].

In the second case, the late 3D universe is considered as filled by a twoconstituent fluid where matter is replaced by radiation (with $p_{r}=\frac{1}{3} \rho_{r}$ ) and a equation of state known as the Chaplygin equation to model the contributions of the dark energy (this was proposed for $4 \mathrm{D}$ models in [7]). Again, the interaction between the constituents is considered to occur via the gravitational field. The Chaplygin equation of state reads

$$
p_{c}=-\frac{A}{\rho_{c}},
$$

where $A$ is a positive parameter. This equation of state would correspond to the phenomenological modelling of a gas of membranes [7. The Einstein and conservation-law equations give in this case

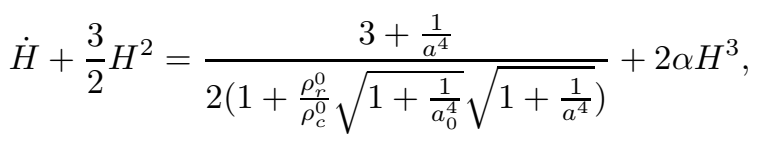




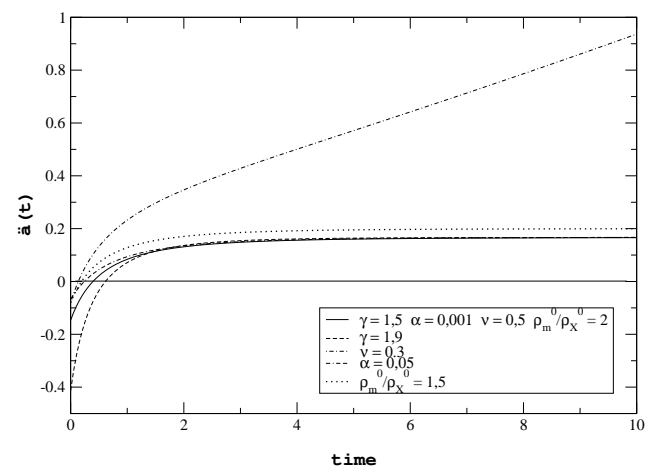

Figure 5: evolution in time of acceleration during the expansion in the dark energy dominated regime

$$
\frac{\rho_{c}}{\rho_{c}^{0}}=\frac{\sqrt{1+\frac{1}{a^{4}}}}{\sqrt{1+\frac{1}{a_{0}^{4}}}}, \frac{\rho_{r}}{\rho_{r}^{0}}=\frac{\rho_{c}^{0}}{\rho_{r}^{0}}\left[\left(1+\frac{\rho_{r}^{0}}{\rho_{c}^{0}}\right) H^{2}-\frac{\sqrt{1+\frac{1}{a^{4}}}}{\sqrt{1+\frac{1}{a_{0}^{4}}}}\right] .
$$

The initial conditions in this case include $a(0)=1, \dot{a}(t)=1$, and $\frac{\rho_{c_{0}}}{\rho_{r_{0}}}=0.1$. The results of the numerical solutions of this system is shown in figure (7).

This shows the acceleration behavior and is easily recognized as a transition to positive values in later times. An increasing value of the viscosity parameter provide a more drastic transition to the accelerated regime. The energy densities behave in a similar way as the scalar field case (see figure (6)). Putting $A=0$ the solutions confirm that the Chaplygin gas is responsible for the positive accelerated regime. On the other hand the scale factor behavior correspond to a never ending expansion. Qualitatively, these results indicate a strong similarity with the ones showed in the last section: both the Chaplygin equation of state (see figure (7)) and the scalar field formulation give a smooth transition to an accelerated regime, dominated by the dark energy constituent, coming from a precedent universe where matter/radiation were predominant.

\section{$5 \quad$ Scalar gravity for 3D universes}

The verification of the singular features of 3D gravity embraces the possibility of testing other models of gravitation in 3D space-times. Focusing on our purposes we mention that the so-called scalar gravity model ( $R=T$ ") was analyzed in a cosmological context in $[3$. A remarkable feature of this model is that, contrary to the 3D Einstein case, its dynamics contains a Newtonian limit 3 . On the other hand, in principle, this model gives an incomplete information about the evolution of the gravitational field [3. However, when we impose the Robertson- 

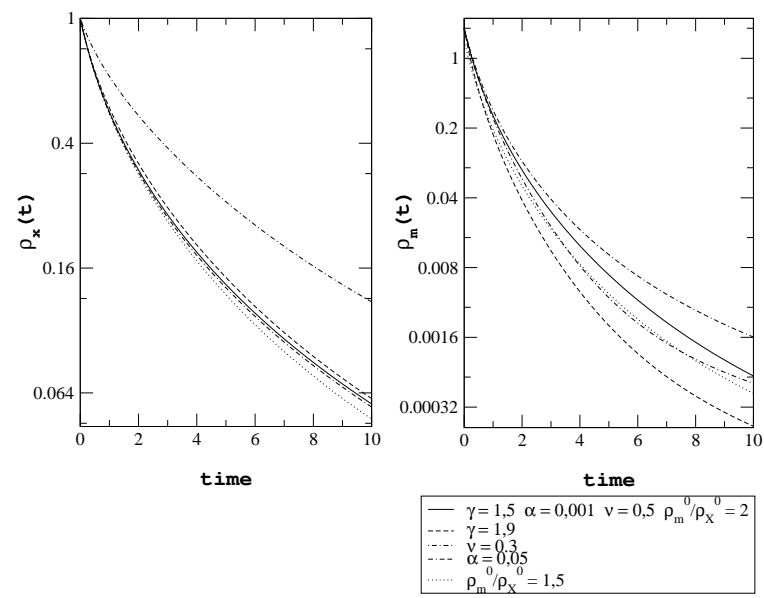

Figure 6: evolution in time of energy densities during the expansion in the dark energy dominated regime

Walker hypothesis different cosmological scenarios can be consistently described; in fact several regimes involving matter and/or radiation were considered in $[3$. In this section we would like to extend those results investigating the behavior of both young and old universes filled by different combinations of constituents (including the inflaton and the dark energy) ruled by a 3D scalar cosmology.

The simplest case possible is to consider a one-constituent model simulating, for example, radiation/matter dominated periods. This was done in [3] with interesting results. The dynamics is ruled by

$$
2 \frac{\ddot{a}}{a}=-\left(\frac{\dot{a}}{a}\right)^{2}+\kappa(2 p-\rho)+\lambda,
$$

where $\lambda$ is a cosmological constant. Our analysis focuses again the possibility of describing transition eras, in particular the end of an inflationary period. Inspired by the results obtained in 2D and 4D models we consider a one-constituent universe ruled by the van der Waals equations of state 8 .

The use of this equation of state in a cosmological context was proposed by [8] explored in [9] and tested in 2D models in [6]. In our case we have as the dynamical equations

$$
2 \frac{\ddot{a}}{a}=-\kappa(\rho-2 p)-\left(\frac{\dot{a}}{a}\right)^{2}+\lambda, \quad \dot{\rho}+2 \frac{\dot{a}}{a}(\rho+p)=0,
$$

coupled to the vdW equation

$$
p=\frac{g \rho}{1-\alpha \rho} .
$$




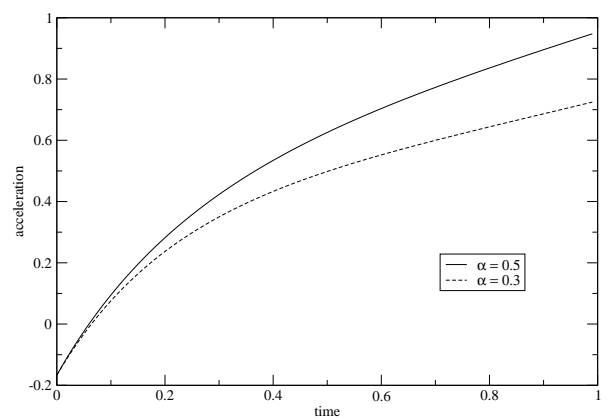

Figure 7: acceleration with dark energy ruled by the Chaplygin equation, for different values of the viscosity parameter

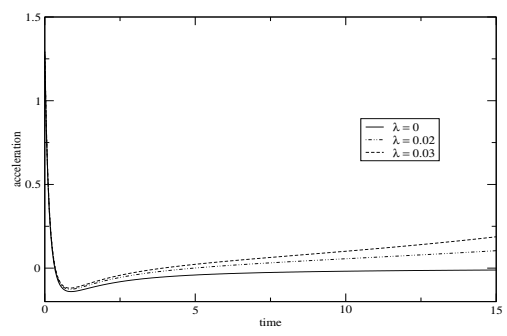

Figure 8: acceleration for different values of cosmological constant $\lambda$

This vdW equation without the quadratic term in $\rho$ was used by Christmann et al. (see ref. [6]) to investigate 2D Robertson-Walker cosmologies. We are taking as initial (normalized) values $\rho_{0}=0.7, a(0)=1, \dot{a}(0)=1$ and typical values for the vdW parameters $g=0.9, \alpha=0.5$. The one-constituent cosmological fluid ruled by the vdW equation can simulate the behavior of a young universe leaving an initial accelerated era as it is shown in figure (8). The results also show that if we include a cosmological constant a three period evolution is possible, with the initial accelerated era followed by decelerated and finally accelerated periods. The behavior of the scale factor says that the universe is in an ever expanding regime, like in all the previous cases. The evolution of the vdW energy density is in tune with the rate of expansion of the scale factor (see figure (9)). As as final remark we confirm that the three-eras regime is possible for particular values of the parameters like those reported above; there are other situations (like in the case when the normalized initial energy density value is lower than 0.5) that a permanent decelerated evolution appears (with the cosmological constant equal to zero), showing that a cosmic fluid obeying the vdW equation does not imply necessarily into an initially accelerated 3D universe. 


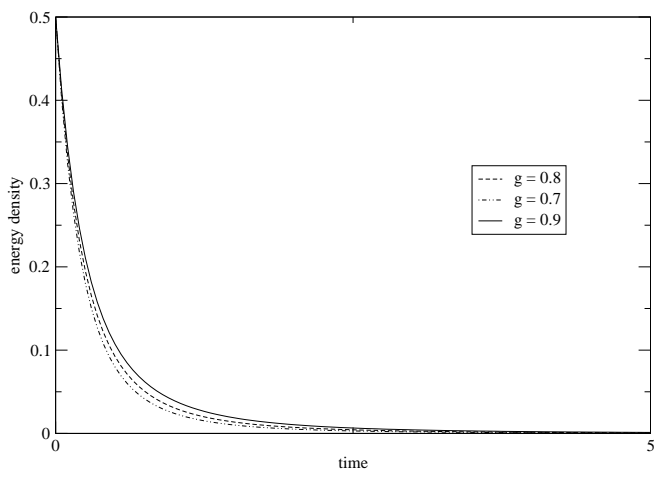

Figure 9: evolution in time of vdW energy density for different values of the barotropic parameter in scalar 3D cosmology

\section{Conclusions}

In this work we have investigated the presence of accelerated regimes, and their transitions to decelerated eras, in 3D cosmological models. Two-constituents scenarios are enough to simulate a young universe leaving the inflationary period (with the inflaton represented by the scalar field) or an old universe coming into a dark energy dominated period; the effects of the dark energy constituent can be also simulated by the inclusion of the Chaplygin equation of state. The alternative scalar model, with the cosmological fluid ruled by the van der Waals equation of state, permits a three-eras evolution when a cosmological constant term is present.

\section{References}

[1] J.D. Brown, Lower dimensional gravity, World Scientific, Singapore, 1993; D. Grumiller et al., Phys. Rept. 369, 327(2002).

[2] S. Giddings,J. Abbott and K. Kuchar, Gen. Rel. Grav. 16, 8 (1983); J.D. Barrow, A.B. Burd, D. Lancaster, Class. Quant. Grav. 3, 551 (1986).

[3] N.J. Cornish and N.E. Frankel, Phys. Rev. D 43, 2555 (1991).

[4] R. Jackiw, Nucl. Phys. B 252, 343 (1985).

[5] W. Zimdahl and D. Pavón, Gen. Rel. Grav. 33, 791 (2001); I. Prigogine, J. Geheniau, E. Gunzig, P. Nardone, Gen. Rel. Grav. 21, 8 (1989); Kremer, G. M. and Devecchi, F. P. Phys. Rev. D 67, 047301 (2003).

[6] R. B. Mann and S. F. Ross, Phys. Rev. D 47, 3312 (1993); M. Cadoni and S. Mignemi, Gen. Rel. Grav. 34, 2101 (2002); M.H. Christmann, F.P. Devecchi, G.M. Kremer, and C.M. Zanetti, Europhys. Lett. 61, 15(2004). 
[7] A. Kamernshchik, U. Moschella and V. Pasquier, Phys. Lett. B 511, 265 (2001); G.M. Kremer, Gen. Rel. Grav. 35, 1459 (2003).

[8] S. Capozziello, S. Martino, M. Falanga, Phys. Lett. A 299,494 (2002).

[9] G.M. Kremer. Phys. Rev. D 68, 123507 (2003).

[10] P.J.E. Peebles, Principles of Physical Cosmology, Princeton University Press, Princeton, 1993. 